\title{
Evaluation of 153 Asymptomatic Subjects Using the Anopress Portable Anal Manometry Device
}

\author{
Cosimo Alex Leo, ${ }^{1,2 *}$ Emanuel Cavazzoni, ${ }^{3}$ Gregory P Thomas, ${ }^{1}$ Jonathan Hodgkison, ${ }^{1,2}$ Jamie Murphy, ${ }^{2}$ and Carolynne J Vaizey ${ }^{1,2}$ \\ ${ }^{1}$ London North West NHS Trust - St Mark's Hospital Academic Institute, Sir Alan Park's Physiology Unit, Harrow, UK; ${ }^{2}$ Imperial College of \\ London, UK; and ${ }^{3}$ Santa Maria della Misericordia Hospital, University of Perugia, Perugia, Italy
}

\section{Background/Aims}

The Anopress device is a new portable manometry system. The aim of this study is to formulate normative data using this new device by recording the anorectal function of asymptomatic subjects. Patient comfort was also assessed.

\section{Methods}

Anorectal function was assessed in asymptomatic volunteers using the Anopress. All volunteers were examined in a standardized way in accordance with the study protocol. Normative values for the Anopress were obtained from the recorded data and patient comfort was assessed using a visual analogue scale.

\section{Results}

We recruited 153 healthy volunteers. Eighty were female (23 parous; median age 39.5 [interquartile range $\{$ IQR $\}, 28.75-53.00]$ ) and 73 were male (median age 40.5 [IQR, 29.00-52.25]). For the female cohort, the following normal range (2.5-97.5 percentile) values were recorded across the whole anal canal: resting pressure 40.0-103.0 mmHg; squeeze increment 35.0-140.6 mmHg; endurance 1.3-9.0 seconds; involuntary squeeze 41.1-120.8 mmHg; and strain pressure 22.1-77.9 mmHg. Similarly, the following male normal range (2.5-97.5 percentile) values were recorded across the whole anal canal: resting pressure 38.3-99.6 mmHg; squeeze increment 42.5-154.8 mmHg; involuntary squeeze $40.0-123.6 \mathrm{mmHg}$; endurance $2.0-10.0$ seconds; and strain pressure $11.0-72.1 \mathrm{mmHg}$. The median visual analogue scale scores for discomfort during the measurement was $0.0(\mathrm{IQR}, 0.00-0.00)$.

\section{Conclusions}

Normative values for the Anopress device have been calculated by this study. The Anopress appears to be a safe and well tolerated way of measuring pressures from the entire anal canal. Further comparisons with other standard and commonly used manometry tests are, however, required to verify its reliability.

(J Neurogastroenterol Motil 2018;24:431-436)

\section{Key Words}

Anal canal; Manometry; Physiology; Pressure

Received: November 24, 2017 Revised: February 5, 2018 Accepted: February 9, 2018

(a) This is an Open Access article distributed under the terms of the Creative Commons Attribution Non-Commercial License (http://creativecommons. org/licenses/by-nc/4.0) which permits unrestricted non-commercial use, distribution, and reproduction in any medium, provided the original work is properly cited.

*Correspondence: Cosimo Alex Leo, MBBS, MD

St Mark's Hospital Academic Institute, 1, Watford Road HA1 3UJ, Middlesex, Harrow, UK Tel: +44-020-8235-4046 (4048), Fax: +44-020-8235-4046 (4048), E-mail: a.leo@nhs.net 


\section{Introduction}

Anorectal physiological testing (ARP) investigates the function of the anorectum, providing diagnostic and prognostic information in a range of anorectal pathologies. ${ }^{1-5}$ Manometric assessment is a key part of ARP and allows estimation of anal canal pressures, which are directly related to anal sphincter structure and function. Measurements can include the anal canal resting pressure, maximum voluntary squeeze increment, involuntary squeeze increment, endurance and strain pressures. ${ }^{1,6,7}$

In recent years, there has been some debate as to which type of device should be used to perform ARP measurements, with discussions focusing on improving the accuracy of ARP assessment. ${ }^{8,9}$ Conventional manometry uses a multi-channel water perfused catheter, which records along both its longitudinal and radial axes. These catheters are durable, require little maintenance and are reliable. Developments in technology, however, have produced high resolution solid state catheters and 3 dimensional high definition manometry. ${ }^{8,11-13}$ These devices have a greater number of sensors per surface area, when compared with water perfused catheters. An additional benefit of this technology is that the topographical display produced by these newer devices may aid interpretation of ARP data. The main limitations of traditional solid state catheters have been their cost and fragility. ${ }^{12-14}$

Anopress (THD Worldwide, Correggio (RE), Italy) is a new portable anal manometry device (Fig. 1), which uses a solid state catheter to evaluate the sphincter pressures generated from the whole of the anal canal. It has European Conformity and US Food and Drug Administration approval. ${ }^{15}$ It has been promoted as a small, portable, wireless, and reliable device that can perform rapid manometric assessments outside the anorectal physiology labora- tory. ${ }^{11,16}$ The aim of this study was to produce normative values for this new device by assessing a group of asymptomatic volunteers. Patient discomfort was also assessed using a visual analogue scale (VAS).

\section{Materials and Methods}

The Anopress device was used to assess 153 asymptomatic volunteers. All subjects included in this study were recruited from the same center. Institutional Review Board approval was granted (247/17; Modena Research Ethics Committee, University of Modena and Reggio Emilia, Italy). Written consent was gained prior to subjects being included in the study. A full clinical history was taken prior to anorectal assessment. All subjects were screened for gastrointestinal symptoms and those who had any history of incontinence, constipation, anal pain, irritable bowel syndrome or inflammatory bowel disease were excluded. Also according to the protocol for this study all the subjects $<18$ and $>80$ year old were excluded.

The procedure was performed by an experienced physician with a specialist interest in anorectal manometry and physiology. All subjects were instructed to defecate if required prior to the procedure and no bowel preparation was given. It was confirmed that all subjects understood the commands squeeze, cough, and push prior to the procedure. ${ }^{17,18}$ Both genders were included in the study and the female cohort was subcategorized into nulliparous and parous groups. Anal resting, voluntary, involuntary, endurance, and strain pressures were determined by following the study protocol (Fig. 2). The data generated by the procedure were automatically recorded and immediately available for review. All the values expressed by Anopress are summarized in Table 1. A VAS score with the descriptor extremes " $0=$ no pain at all" and " $10=$ my pain is as
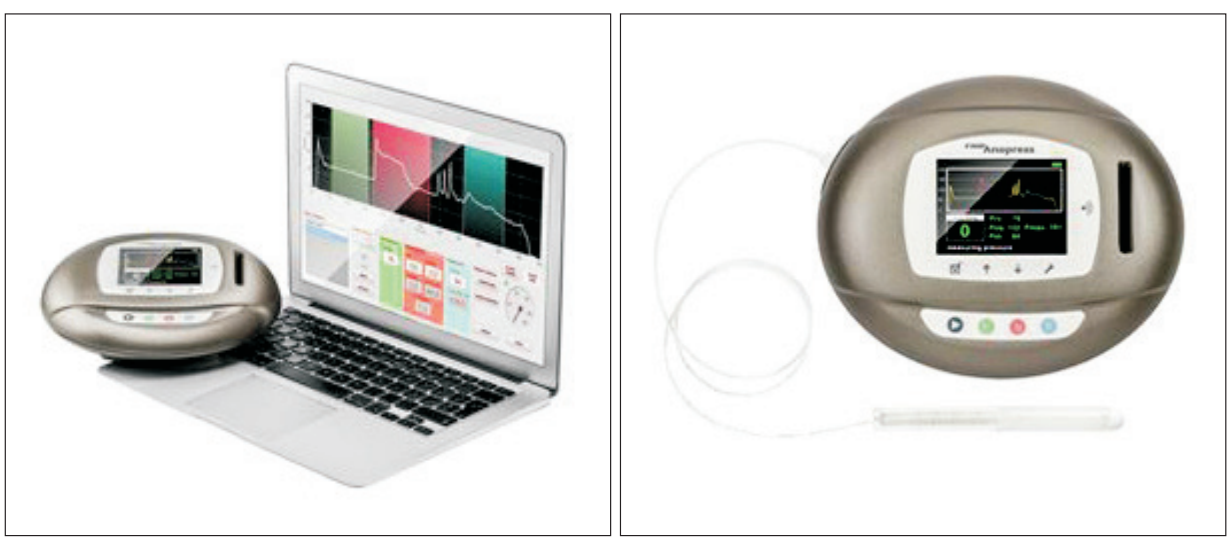

Figure 1. The Anopress device is shown here with a laptop computer and a solid state catheter connected. Bluetooth technology allows the Anopress device to work wirelessly. 
bad as it could possibly be" was used to evaluate how comfortable patients found this new device. ${ }^{19}$ Data are presented using either median values with interquartile range (IQR; 25 th-75th percentile) or normal range (2.5-97.5 percentile). ${ }^{20}$ Statistical analyses were performed using commercially available software (GraphPad Prism, Software Version 6, La Jolla, CA, USA). A P-value $<0.05$ was considered statistically significant.

\section{The Technique (see Supplementary Video)}

The Anopress device can communicate with any laptop com-

\begin{tabular}{|c|}
\hline 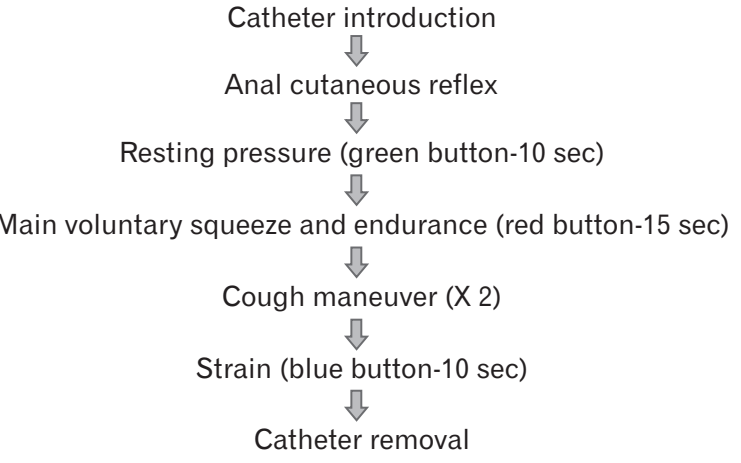 \\
\hline
\end{tabular}

Fiğure 2. Study protocol for Anopress device: assessment of asymptomatic volunteers. puter via a Bluetooth connection. The solid state catheter provided with the device must first be connected. This is achieved by rotating the catheter clockwise into the Anopress device connector. The catheters are made from latex free PVC, are $9 \mathrm{~mm}$ in diameter, and have a single transduction area $5 \mathrm{~cm}$ in length on their radial surface. The transduction area is an air filled space which transmits any pressure modification imposed onto the catheter. These pressure modifications are recorded every tenth of a second for the duration of the examination. The device is calibrated by pushing the start button; this phase of the procedure takes approximately 10 seconds. The patient is positioned on the examination couch in the standard left lateral position. The catheter is lubricated and inserted gently into the anal canal until the area of the probe is in contact with the entire surface of the anal canal. The probe records the real time mean pressure of that entire area, which represents the radial forces generated by the whole sphincter complex. The device has 3 buttons that record specific phases of the procedure: resting pressure (green button), squeeze pressure (red button), and strain pressure (blue button). Subjects are initially asked to relax to allow the anal resting pressure to be measured until a stable trace is achieved which is then recorded for 10 seconds. Following this, the patient is asked to squeeze as hard and long as possible to measure the maximum anal squeeze pressure and the anal endurance pressure over a period of 15 seconds. Involuntary squeeze pressures are measured by asking the patient to cough. Finally, subjects are asked to bear down, or to

Table 1. All the Values Expressed by Anopress. These Can Be Visualized in the Main Screen and Easily Interpreted

Anal cutaneous reflex: initial pressure peak that represents the guard anal reflex.

Resting pressure: pressure wave with a steady and constant development, without significant peaks. This is the expression of the pressure of the internal sphincter at rest.

Maximum voluntary squeeze: the highest recorded pressure during the squeeze maneuver.

Squeeze increment: the maximum highest recorded pressure minus the main resting pressure. This pressure is also recorded during the squeeze maneuver.

Endurance (expressed in seconds): reflecting the length of a voluntary contraction of the external sphincter for the time that it exceed the $50 \%$ of the maximum squeeze/maximum rest pressure rate.

Mean 10 seconds squeeze increment: the increasing of average pressure within 10 seconds of maximum squeeze.

Cough squeeze (involuntary squeeze): the maximum squeeze recorded during the cough maneuver.

Strain pressure: wave the normally slightly increase at first and then decreases and stabiles to the resting pressure values. This happens during the push maneuver which simulate the defecation.

(Max/Rest) Maximum pressure/resting pressure rate (expressed in folds): describes how many times the top squeeze pressure exceed the mean resting pressure.

(\%Avg/Max) Percentage of mean resting pressure/mean strain pressure: reflects the percentage of increase or reduction of pressure during the whole phase of strain, compared to the mean resting pressure.

(\%Avg/Rest Avg) The mean strain/mean resting percentage rate: is the comparison between the pressure at rest and the pressure during the attempted defecation phase. The negativity of the percentage is seen in normal individuals, when the pressure during strain is lower than during the rest phase, expressing a relaxation of the muscular complex. A positive percentage, especially above $20 \%$, can be seen in individuals with paradoxical contraction of the external sphincter during defecation, and is common in patients complaining with obstructed defecation syndrome. 

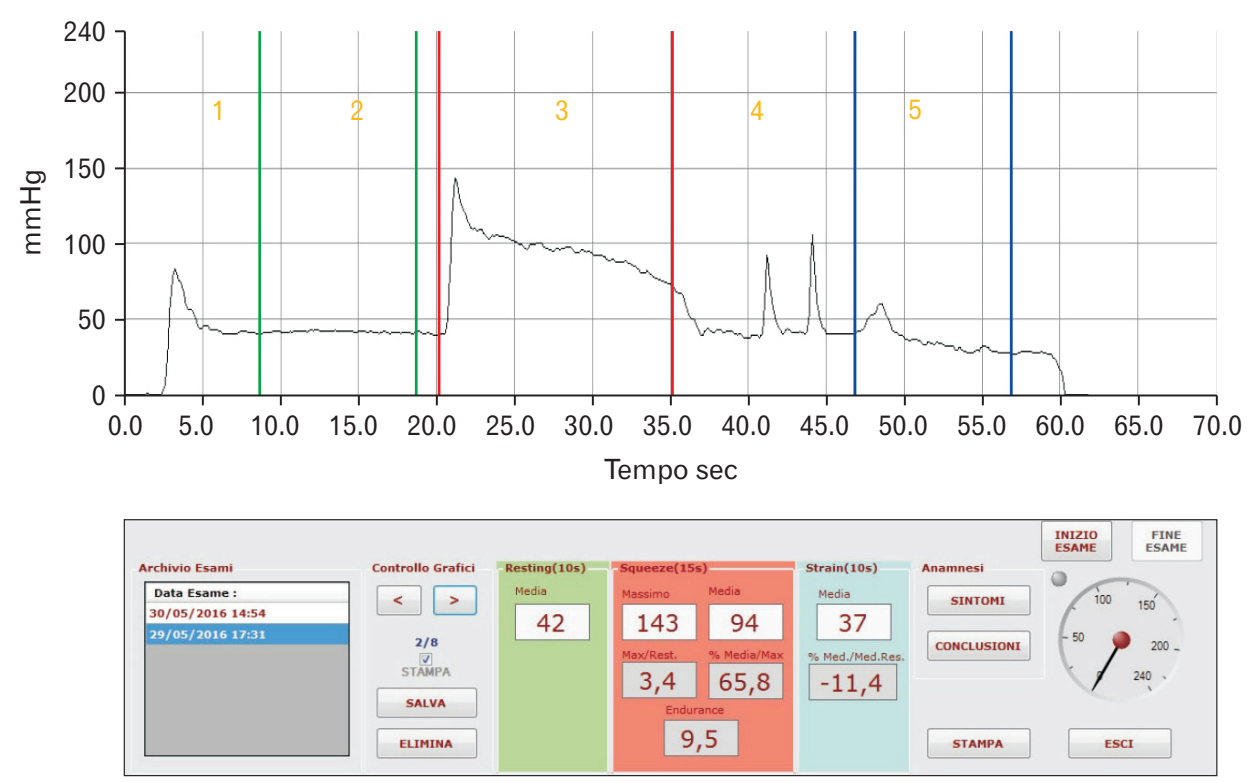

Figure 3. Results from an Anopress manometric study in an asymptomatic volunteer. The graph follows the protocol described above. strain for 10 seconds, in order to simulate defecation (Fig. 2 and 3). At this point, the catheter is removed and the data generated by the procedure are immediately available for analysis using the laptop computer and can be edited accordingly.

\section{Results}

Between April 2016 and June 2017, 153 asymptomatic subjects were recruited, $80(53.3 \%)$ of whom were female. The median age was 39.5 (IQR, 28.75-53.00) and 40.5 (IQR, 29.00-52.25) years of age in the female and male groups, respectively. Of the female cohort, 23 were parous and the median age was 40 (range, 19-79).

Normal range (2.5-97.5 percentile) values were recorded across the whole anal canal for both female and male patients. The calculated normal anal resting pressure ranges were 40.0-103.0 $\mathrm{mmHg}$ in the female group and 38.3-99.6 $\mathrm{mmHg}$ in the male group. Normal range values for maximum squeeze increment were 35.0-140.6 $\mathrm{mmHg}$ in the female and $42.5-154.8 \mathrm{mmHg}$ in the male subject groups. Normal values for endurance squeeze were calculated as 1.3-9.0 seconds in the female and 2.0-10.0 seconds in the male groups. The normal range for involuntary maximum squeeze was 41.1-120.8 $\mathrm{mmHg}$ in the female group and 40.0-123.6 $\mathrm{mmHg}$ in the male group. Finally, normal range strain pressures were 22.1$77.9 \mathrm{mmHg}$ and $11.0-72.1 \mathrm{mmHg}$ respectively in the female and male cohorts. Normal range values for Anopress assessment in all groups are summarized in Table 2 .

When the male and female groups were compared, a statistically significant difference was noted for anal resting pressure $(P$
$=0.044)$, squeeze increment $(P<0.001)$ and endurance squeeze pressures $(P=0.042)$. When nulliparous and parous female cohorts were compared, a statistically significant difference was noted for anal resting pressure $(P=0.026)$. Results of the statistical analysis are outlined in Table 2.

All subjects tolerated the procedure without significant discomfort and a median VAS score of 0.0 (IQR, 0.00-0.00) was recorded for all study subjects. No complications occurred during the study. In all cases the probe was placed once and did not need to be repositioned for the appropriate measurements to be taken.

\section{Discussion}

This study was designed to obtain the first normative values for the new Anopress manometry device, which have been presented above. To the best of the authors' knowledge, this study is also one of the largest reported groups in the published literature describing normal values for anal manometry in asymptomatic subjects. Assessment using VAS revealed patients found Anopress to be acceptable, with only few subjects reporting a minimal discomfort during testing. While anal manometry recordings were performed rapidly and were well tolerated when using this device, the current commercially available catheter cannot measure anal canal length or the recto-anal inhibitory reflex; however, these limitations will be overcome with the next iteration of this device.

The subjects recruited to this study were a heterogeneous cohort of male and female (both nulliparous and parous) subjects. This heterogeneity was explicitly sought in order to generate normal 
Table 2. Summary of Normal Values for Measuring Anorectal Function Using Anopress in Asymptomatic Volunteers (A) Normal Values Measured by Anopress in Asymptomatic Male and Female Volunteers

\begin{tabular}{|c|c|c|c|c|c|}
\hline & \multicolumn{4}{|c|}{ Asymptomatic volunteers $(\mathrm{n}=153)$} & \multirow{3}{*}{$P$-value } \\
\hline & \multicolumn{2}{|c|}{ Female $(\mathrm{n}=80)$} & \multicolumn{2}{|c|}{ Male $(\mathrm{n}=73)$} & \\
\hline & Median (IQR) & Normal range & Median (IQR) & Normal range & \\
\hline Resting pressure (mmHg) & $67(54.8-79.0)$ & $40.0-103.0$ & $61.8(54.8-79.0)$ & $38.3-99.6$ & $0.044^{\mathrm{a}}$ \\
\hline Squeeze increment $(\mathrm{mmHg})$ & $75.5(54.5-98.0)$ & $35.0-140.6$ & $101.0(86.0-121.0)$ & $42.5-154.8$ & $<0.001^{\mathrm{a}}$ \\
\hline Involuntary squeeze ( $\mathrm{mmHg}$ ) & $69.5(55.0-98.0)$ & $41.1-120.8$ & $79(56.0-99.0)$ & $40.0-123.6$ & 0.236 \\
\hline Endurance (sec) & $4.5(4.0-5.1)$ & $1.3-9.0$ & $5.0(4.0-6.3)$ & $2.0-10.0$ & $0.042^{\mathrm{a}}$ \\
\hline Mean 10 sec squeeze increment $(\mathrm{mmHg})$ & $61.0(54.0-72.0)$ & $44.0-98.0$ & $60.0(54.5-77.0)$ & $43.0-103.0$ & 0.240 \\
\hline Strain $(\mathrm{mmHg})$ & $43.5(31.0-54.3)$ & $22.1-77.9$ & $45.2(32.0-55.0)$ & $11.0-72.1$ & 0.564 \\
\hline
\end{tabular}

(B) The Difference of Normal Values Measured by Anopress Between Parous and Nulliparous Female Group

\begin{tabular}{|c|c|c|c|c|c|}
\hline & \multicolumn{4}{|c|}{ Female asymptomatic volunteers $(\mathrm{n}=80)$} & \multirow{3}{*}{$P$-value } \\
\hline & \multicolumn{2}{|c|}{ Nulliparous $(\mathrm{n}=57)$} & \multicolumn{2}{|c|}{ Parous $(n=23)$} & \\
\hline & Median (IQR) & Normal range & Median (IQR) & Normal range & \\
\hline Resting pressure (mmHg) & $61.0(54.0-78.0)$ & $39.5-102.1$ & $75.0(67.0-84.5)$ & $41.0-105.0$ & $0.026^{\mathrm{a}}$ \\
\hline Squeeze increment $(\mathrm{mmHg})$ & $75.0(57.0-95.0)$ & $30.5-137.0$ & $83(53.0-102.0)$ & $35.0-141.0$ & 0.468 \\
\hline Involuntary squeeze $(\mathrm{mmHg})$ & $76.0(55.0-98.0)$ & $41.0-135.3$ & $65.0(55.0-87.0)$ & $45.0-110.0$ & 0.311 \\
\hline Endurance (sec) & $4.5(4.0-5.1)$ & $1.2-9.1$ & $4.1(3.8-5.1)$ & $2.9-7.0$ & 0.464 \\
\hline Strain $(\mathrm{mmHg})$ & $41.0(31.0-54.0)$ & $23.4-75.3$ & $50.0(43.0-55.5)$ & $21.0-78.0$ & 0.053 \\
\hline
\end{tabular}

All the pressures are expressed using either median values with interquartile range (IQR; 25th-75th percentile) and normal range in 2.5 and 97.5 percentile.

Mann-Whitney $\mathrm{U}$ analysis was used to analyse differences between male and female groups and between nulliparous and parous female groups ( ${ }^{\mathrm{a}} P$-value $\left.<0.05\right)$.

values that would be applicable for disparate groups of patients in future. As expected and as already reported by previous studies for different manometry devices, we observed differences between the subgroups of our study cohort. ${ }^{7,10,21,22}$ Specifically we observed significant differences in resting pressures, squeeze increment and endurance pressures when comparing male and female subjects, contributed to by the longer anal canal in men. ${ }^{10,21-25}$ The significant difference seen in resting pressures noted when comparing parous and nulliparous women is likely to be a consequence of unidentified and asymptomatic sphincteric lesions caused by obstetric injury. In our opinion, the nulliparous female group should be considered the true normative values, as the parous group are potentially an asymptomatic cohort at present who may go on to develop functional problems in future, rather than a group of truly asymptomatic healthy volunteers

When comparing our results with the published literature the normative values for Anopress are lower than values used for water perfused manometry and previously validated high resolution manometry values. ${ }^{10}$ Our data are in keeping with previous published reports assessing another portable manometry device (Mcompass; Medspira, Minneapolis, MN, USA $)^{10,14}$; However, published data assessing this device are limited given one of these studies assessed only a small cohort as the authors aim was to validate pressure measurements rather than report normative values. The lower normal values reported by our study and those assessing the Medspira device are due to the technologies used by these portable devices, which record values from the whole of the anal canal rather than the maximum values along the length of the canal as is the case for other devices.

In conclusion, normal range values for Anopress have been reported by this study. The Anopress device appears to be well tolerated, is portable, and has the advantage that it can be used at the bedside by any physician. While the current catheter technology allows assessment of the entire anal canal it is limited by its inability to record anal canal length or be used to elicit the recto-anal inhibitory reflex. These limitations will be addressed in the next iteration of this technology. Further comparisons with other standard and commonly used manometry systems will be required in future to evaluate the reliability of the Anopress device.

\section{Supplementary Material}

Note: To access the supplementary video mentioned in this article, visit the online version of Journal of Neurogastroenterol- 
ogy and Motility at http://www.jnmjournal.org/, and at https://doi. org/10.5056/jnm17135.

Acknowledgements: We thank THD SpA for providing the Anopress device.

\section{Financial support: None.}

\section{Conflicts of interest: None.}

Author contributions: Cosimo Alex Leo and Emanuel Cavazzoni have contributed to design of the study, acquisition of data, analysis and interpretation of data, and drafting/revising the manuscript critically for important intellectual content; Jonathan Hodgkison, Jamie Murphy, and Gregory P Thomas have contributed to the design and interpretation of data and revising the manuscript; Carolynne J Vaizey has contributed to the conception and design, acquisition of data, analysis and interpretation of data, drafting/ revising the manuscript critically for important intellectual content; and all authors contributed provided a final approval of the version to be published.

\section{References}

1. Meunier PD, Gallavardin D. Anorectal manometry: the state of the art. Dig Dis 1993;11:252-264.

2. Rao SS, Azpiroz F, Diamant N, Enck P, Tougas G, Wald A. Minimum standards of anorectal manometry. Neurogastroenterol Motil 2002;14:553-559.

3. Fabrizio AC, Alimi Y, Kumar AS. Methods of evaluation of anorectal causes of obstructed defecation. Clin Colon Rectal Surg 2017;30:46-56.

4. Pucciani F, Ringressi MN. Obstructed defecation: the role of anorectal manometry. Tech Coloproctol 2012;16:67-72.

5. Pucciani F. Anorectal manometry. In: Santoro G, Wieczorek AP, Bartram C, eds. Pelvic floor disorders: imaging and multidisciplinary approach to management. Milan: Springer 2010:315-318.

6. Kim JH. How to interpret conventional anorectal manometry. J Neurogastroenterol Motil 2010;16:437-439.

7. Cali RL, Blatchford GJ, Perry RE, Pitsch RM, Thorson AG, Christensen MA. Normal variation in anorectal manometry. Dis Colon Rectum 1992;35:1161-1164.

8. Lee TH, Bharucha AE. How to perform and interpret a high-resolution anorectal manometry test. J Neurogastroenterol Motil 2016;22:46-59.

9. Carrington EV, Grossi U, Knowles CH, Scott SM. Normal values for high-resolution anorectal manometry: a time for consensus and collabora- tion. Neurogastroenterol Motil 2014;26:1356-1357.

10. Carrington EV, Brokjaer A, Craven H, et al. Traditional measures of normal anal sphincter function using high-resolution anorectal manometry (HRAM) in 115 healthy volunteers. Neurogastroenterol Motil 2014;26:625-635.

11. Cavazzoni E. Manual of clinical anorectal manometry. 1st ed. Roma: Youcanprint 2017.

12. Rasijeff AMP, Withers M, Burke JM, Jackson W, Scott SM. Highresolution anorectal manometry: a comparison of solid-state and waterperfused catheters. Neurogastroenterol Motil 2017;29:e13124.

13. Ihnát $\mathrm{P}$, Vávra $\mathrm{P}$, Guňková $\mathrm{P}$, Pelikán $\mathrm{A}$, Zonca P. 3D high resolution anorectal manometry in functional anorectal evaluation. Rozhl Chir 2014;93:524-529.

14. Bharucha AE, Stroetz R, Feuerhak K, Szarka LA, Zinsmeister AR. A novel technique for bedside anorectal manometry in humans. Neurogastroenterol Motil 2015;27:1504-1508.

15. U.S. Food and drug administration. Available from URL: https://www. fda.gov/MedicalDevices/default.htm U.S. Food and Drug Administration (accessed 7 June 2018).

16. THD Spa Italy. Available from URL: http://thdanopress.com/ (accessed 7 June 2018).

17. Gonçalves ML, Fernandes SF, de Almeida RM, Diaz FA, de Oliveira PG, de Sousa JB. Anorectal manometry evaluation in adult women with clinical and urodynamic diagnostics of overactive bladder. Arq Bras Cir Dig 2013;26:280-285.

18. Heinrich H, Fruehauf H, Sauter M, et al. The effect of standard compared to enhanced instruction and verbal feedback on anorectal manometry measurements. Neurogastroenterol Motil 2013;25:230-237, e163.

19. Crichton N. Visual analogue scale (VAS). J Clin Nurs 2001;10:706-706.

20. Bland M. An introduction to medical statistics. Third edition. New York: Oxford Medical Publications 1986.

21. Jameson JS, Chia YW, Kamm MA, Speakman CT, Chye YH, Henry MM. Effect of age, sex and parity on anorectal function. Br J Surg 1994;81:1689-1692.

22. Williams AB, Cheetham MJ, Bartram CI, et al. Gender differences in the longitudinal pressure profile of the anal canal related to anatomical structure as demonstrated on three-dimensional anal endosonography. $\mathrm{Br}$ J Surg 2000;87:1674-1679.

23. Lazarescu, A, Sadowski D.C. High resolution anorectal manometry: establishment of normal values in healthy volunteers. Gastroenterology 2011;140:S-796

24. Coss-Adame E, Rao SS, Valestin J, Ali-Azamar A, Remes-Troche JM. Accuracy and reproducibility of high-definition anorectal manometry and pressure topography analyses in healthy subjects. Clin Gastroenterol Hepatol 2015;13:1143-1150, e1.

25. Li Y, Yang X, Xu C, Zhang Y, Zhang X. Normal values and pressure morphology for three-dimensional high-resolution anorectal manometry of asymptomatic adults: a study in 110 subjects. Int J Colorectal Dis 2013;28:1161-1168 